\title{
Characterization of unidirectional carbon fiber reinforced polyamide- 6 thermoplastic composite under longitudinal compression loading at high strain rate
}

\author{
Marina Ploeckl ${ }^{\mathrm{a}}$, Peter Kuhn, and Hannes Koerber \\ Institute for Carbon Composites, Technische Universitaet Muenchen, Faculty of Mechanical Engineering, \\ Boltzmannstraße 15, 85748 Garching bei Muenchen, Germany
}

\begin{abstract}
In the presented work, an experimental investigation has been performed to characterize the strain rate dependency of unidirectional carbon fiber reinforced polyamide-6 composite for longitudinal compression loading. An end-loaded compression specimen geometry, suitable for contactless optical strain measurement via digital image correlation and dynamic loading in a split-Hopkinson pressure bar, was developed. For the dynamic experiments at a constant strain rate of $100 \mathrm{~s}^{-1}$ a modified version of the Dynamic Compression Fixture, developed by Koerber and Camanho [Koerber and Camanho, Composites Part A, 42, $462-470,2011]$ was used. The results were compared with quasi-static test results at a strain rate of $3 \cdot 10^{-4} \mathrm{~s}^{-1}$ using the same specimen geometry. It was found that the longitudinal compressive strength increased by $61 \%$ compared to the strength value obtained from the quasi-static tests.
\end{abstract}

\section{Introduction}

Carbon fiber reinforced thermoplastic matrix composites are becoming increasingly attractive for automotive structural applications due to advantages in processing, damage tolerance and recyclability compared to conventional composites with thermoset resin systems. The design of composite parts for modern cars with corresponding safety precautions has to take into account high strain rate loading conditions especially for primary structures. In addition, finite-element simulations of impact and crash scenarios require dynamic material data to ensure a faithful reproduction of the mechanical response.

In this study the focus of the material characterization is on the unidirectional carbon fiber reinforced polyamide-6 (PA 6) composite material. This semicrystalline thermoplastic polymer is one of the most commonly used polymers and currently receives much attention as a matrix material for future automotive composite structures. Common material properties of polyamide- 6 and the quasi-static mechanical properties of short glass fiber reinforced polyamide- 6 are well documented [1-3].

Walley and Field studied the strain rate sensitivity of the uniaxial compressive stress-strain response of moist and dry polyamide- 6 using a direct impact Hopkinson bar (DIHB) [4]. The maximum stress increased with increasing loading rate. Similar results for the compressive [5] and tensile [5-8] behavior of the pure polymer under dynamic loading were investigated. Also the tensile strength of short and long glass fiber reinforced polyamide was found to increase with strain rate [7-11]. To the

${ }^{\mathrm{a}}$ Corresponding author: ploeckl@lcc.mw. tum.de knowledge of the authors only few attempts were carried out to characterize the dynamic stress-strain response of carbon fiber reinforced polyamide- 6 composites. Work done by Todo et al. [12] on plain weave fabric carbon and glass fiber reinforced PA 6 suggested a linear relationship between the tensile strength behavior of the composite and the logarithmic strain rate. A recent study of Jendli et al. [13] shows the characterization of twill woven carbon fiber reinforced polyamide- 66 under $0^{\circ}, 90^{\circ}$ and $\pm 45^{\circ}$ tensile loading from quasi-static to high strain rates. They observed positive strain rate effects on the tensile strength for all loading directions. Both, Todo et al. [12] and Jendli et al. [13] used servo-hydraulic testing machines.

In the above mentioned studies the focus is on the characterization of the strain rate effect of the pure thermoplastic polymer, glass fiber reinforced or woven carbon fiber reinforced PA 6. The present study contributes to the description of the strain rate dependency of a unidirectional carbon fiber reinforced polyamide6 composite. An experimental characterization method, composed of a specific end-loaded specimen geometry and a modified Dynamic Compression Fixture [14], has been developed. The composite material is loaded under longitudinal compression, using an electro-mechanical testing machine for the quasi-static tests and a splitHopkinson pressure bar (SHPB) for the dynamic tests.

The developed specimen geometry offers the possibility to detect the strain-time behavior via digital image correlation. A valid longitudinal compressive failure mode, e.g. failure away from the loading surfaces, was obtained with the developed specimen geometry. This represents an improvement over the earlier study carried out by Koerber and Camanho [14], where the static and dynamic specimens failed at the loading faces. In [14] the measured 
a)

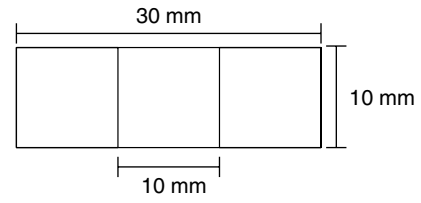

b)

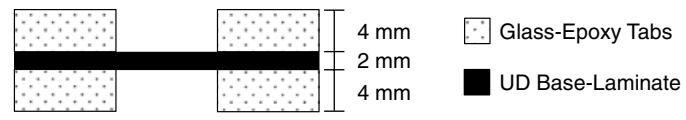

Figure 1. Top view a) and side view b) of end-loaded compression specimen geometry for quasi-static and high strain rate measurements.

strain rate effect on the longitudinal compressive strength therefore may have been somewhat under predicted. In the following text, the static tests performed with the endloaded specimen geometry will be referred to as reference tests, which will be compared to the dynamic test series, using the same end-loaded specimen geometry.

\section{Experimental procedure}

\subsection{Material}

The specimens were prepared from Celstran ${ }^{\circledR}$ CFR-TP PA6 CF60-01, a 60\% carbon fiber by weight polyamide6 unidirectional reinforced thermoplastic composite tape [15]. According to the material data sheet [15] a fiber volume content of $48 \%$ is then obtained. In the present study the fiber volume content was determined to be 49\%, using the test standard ASTM D3171, Method II, Procedure B (digestion of the matrix by a mixture of sulfuric acid and hydrogen peroxide) [16]. This material system is used in industrial, automotive and sporting goods applications and is commonly processed with automated fiber placement technologies. To manufacture 16-ply unidirectional plates, stripes of the tape were laid parallel and brick-like above each other such that the abutting edges of the stripes are at different locations in the individual layers. For a better handling of the stack the frame was welded at a few points with an ultrasonic welding apparatus (ProteUS, EM-Systeme GmbH) with a sonotrode frequency of $30 \mathrm{kHz}$. Using a RUCKS hot-press and a compression mold, the composite plates were consolidated at $260{ }^{\circ} \mathrm{C}$ with $0.5 \mathrm{MPa}$ pressure for 15 minutes.

\subsection{Specimen}

In the presented work, an end-loaded compression specimen geometry suitable for contactless optical strain measurement via digital image correlation (DIC) and dynamic loading in a split-Hopkinson pressure bar was developed (Fig. 1). The specimen consists of a flat rectangular unidirectional (UD) base-laminate with dimensions of $30 \times 10 \times 2 \mathrm{~mm}^{3}$ (length $\times$ width $\times$ thickness). To improve the stability of the specimen and to prevent premature failure at the loading ends, the cross-sectional area at the loading ends was increased by bonding two layers of glass-epoxy composite tabs with the adhesive Scotch-Weld $^{\mathrm{TM}}$ DP 490 from 3M (for the quasistatic test specimens) and BETAMATE ${ }^{\mathrm{TM}} 1822$ from Dow

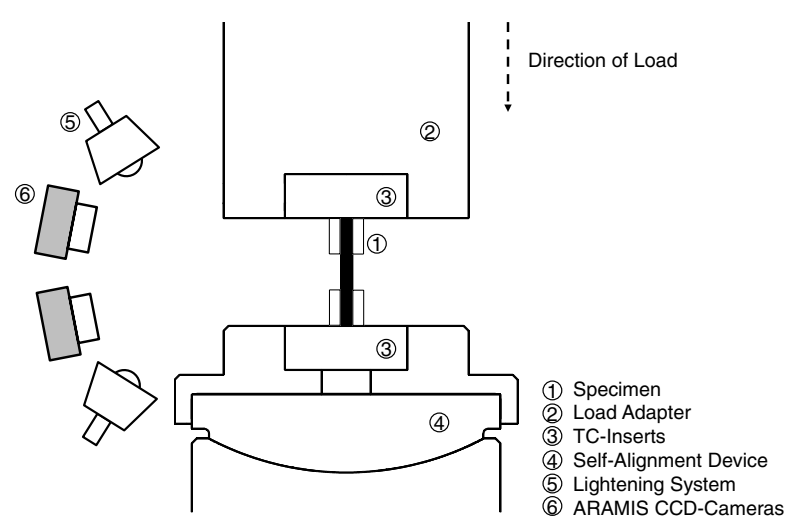

Figure 2. Quasi-static compression test setup with tungstencarbide inserts.

Automotive (for the dynamic test specimens) to both sides, leaving a free gauge section with a length of $10 \mathrm{~mm}$ at the middle of the specimen. The specimens were all cut on a water-cooled diamond saw and the surface parallelism was found to be within $0.02 \mathrm{~mm}$. The loading surfaces were treated with a grinding machine to generate plane and parallel end-faces of good quality. A black-on-withe speckle pattern for the digital image correlation method was applied on the surface of each specimen, using aerosol spray painting. Based on the different image resolutions of the respective cameras the random speckles of the quasistatic specimens were smaller than the speckles of the dynamic specimens.

All specimens were dried in a drying oven under vacuum at a temperature of $80^{\circ} \mathrm{C}$ for 72 hours. The dry specimens were stored in an aluminum multi-layer film bag until start of the experiment to avoid moisture absorption. Before each experiment a thin layer of molybdenum disulfide lubricant was applied on the endsurface of the specimen to reduce friction.

\subsection{Quasi-static test setup}

Quasi-static reference tests were performed for five endloaded compression specimens. An electro-mechanical testing machine of the type Inspekt Table 100 from Hegewald \& Peschke was used with a displacement rate of $0.5 \mathrm{~mm} / \mathrm{min}$, which corresponds to a quasistatic axial strain rate of approximately $3 \cdot 10^{-4} \mathrm{~s}^{-1}$ by considering a nominal specimen length of $30 \mathrm{~mm}$. The load was introduced by means of a self-aligning end-loaded compression setup with embedded polished tungstencarbide inserts at the loading surfaces (Fig. 2). The latter prevent damage on the loading surfaces due to the high compressive strength of the specimen in fiber direction. A $100 \mathrm{kN}$ load cell was used to measure the force. The GOM ARAMIS-4M system, consisting of two CCD cameras with a resolution of $1728 \times 2352$ pixel $^{2}$ and two object lenses of type Titanar $50 \mathrm{~mm}$, were used to obtain the three-dimensional quasi-static strain field of the specimen front surface. The optical strain measurement system was positioned at a distance of $225 \mathrm{~mm}$ away from the specimen surface. The cameras were mounted on a support bar in a distance of $140 \mathrm{~mm}$ to each other and oriented in such a way, that the light paths generate an angle of $25^{\circ}$. 


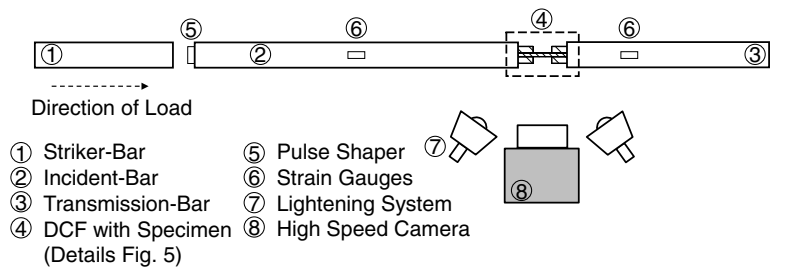

Figure 3. Split-Hopkinson pressure bar test setup with modified Dynamic Compression Fixture (DCF).

To ensure a uniform illumination of the specimen surface two LED lamps beside each camera were used. The acquisition rate of the cameras was set to 5 frames per second (fps) with a shutter speed of $75 \mathrm{~ms}$. For post processing of the recorded pictures the digital image correlation software of the GOM ARAMIS-4M system with a facet size of $17 \times 17$ pixel $^{2}$ and a facet step of $15 \times 15$ pixel $^{2}$ was used. The computation size was set to $3 \times 3$ facets $^{2}$. For the determination of the axial specimen strain, used in the later shown stress-strain curves, the mean value of a $6 \times 6 \mathrm{~mm}^{2}$ strain field at the center of the specimen surface was calculated. The quasi-static longitudinal compressive strength is defined as the quotient of the failure load and the specimen cross-section.

\subsection{Dynamic test setup}

High strain rate tests were performed for five end-loaded compression specimens. A split-Hopkinson pressure bar setup (Fig. 3) was used consisting of diameter $18 \mathrm{~mm}$ steel striker-, incident-, and transmission-bars with lengths of $0.6 \mathrm{~m}, 2.6 \mathrm{~m}$ and $1.6 \mathrm{~m}$, respectively. The strain gauges for the detection of the elastic wave are located on the incident-bar at $1.3 \mathrm{~m}$ and on the transmission-bar at $0.3 \mathrm{~m}$ away from the bar-specimen interfaces and operate with a supply voltage of $7 \mathrm{~V}$. The bar-gauge signals were amplified by using a high speed transducer amplifier of the type FYLDE FE-H379-TA with a gain setting of 100 and a frequency setting of $300 \mathrm{kHz}$. The amplified signals and the impact velocity of the striker-bar were each recorded with a TEKTRONIX TDS2004C oscilloscope. To generate an elastic wave with a triangular shape in the incident bar, a copper pulse shaper with a diameter of $4 \mathrm{~mm}$ and a thickness of $1 \mathrm{~mm}$ was used. The pulse shaper provides a triangular shape which is best suited for specimens exhibiting a linear stress-strain behavior up to failure. For the high strain rate tests an impact velocity of the striker-bar of approximately $7 \mathrm{~m} / \mathrm{s}$ was reached by using an appropriate adjustment of the pressure at the gas gun. As a result of the nominal specimen length of $30 \mathrm{~mm}$ and the striker-bar velocity a strain rate of approximately $100 \mathrm{~s}^{-1}$ was reached.

In the presented work, a modified version of the Dynamic Compression Fixture (DCF), developed by Koerber and Camanho [14] was used. The modified DCF (Fig. 4) consists of two tungsten-carbide (TC) inserts, surrounded by support-rings and placed between the bars and the specimen loading surfaces. Thus, indentation of the bar-ends and stress concentrations at the specimen end-surfaces could be avoided. To prevent an influence of the TC-inserts on the bar strain waves, the mechanical

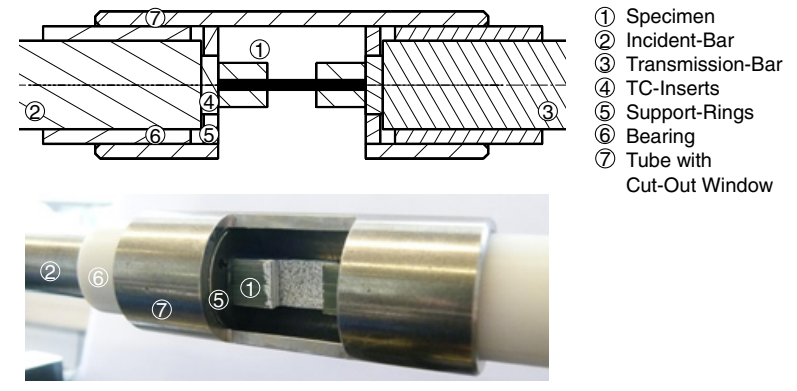

Figure 4. Modified Dynamic Compression Fixture used in the SHPB setup.

impedance between TC-inserts and the Hopkinson bars was matched by choosing an appropriate diameter ratio. The TC-inserts/support-rings are then aligned and held in place by a freely sliding tube, containing a cut-out window to monitor the specimen deformation and failure mode using a high speed camera. The specimen was clamped between the two inserts without any additional fixation.

A high speed camera of type FASTCAM SA 5 from Photron with a ZEISS Makro-Planar T* 2/100 mm ZF lens was positioned $950 \mathrm{~mm}$ away from the specimen surface to capture pictures with a frame rate of $372000 \mathrm{fps}$ at a resolution of $128 \times 80$ pixel $^{2}$ during the experiment. The lens aperture was set to $f / 2.8$. Two spotlights of type DEDOLIGHT 400D were used to provide a well illuminated specimen surface. For post processing of the recorded pictures the digital image correlation software of the GOM ARAMIS-4M system with a facet size of $10 \times 10$ pixel $^{2}$ and a facet step of $8 \times 8$ pixel $^{2}$ was used. The computation size was set to $5 \times 5$ facets $^{2}$. For the determination of the axial specimen strain, the mean value of a $5 \times 5 \mathrm{~mm}^{2}$ strain field at the center of the specimen surface was calculated.

\section{Results and discussion}

\subsection{Quasi-static experimental results}

Five quasi-static reference specimens were loaded until failure at constant displacement rate. The longitudinal compressive stress-strain response for all tested specimens is shown in Fig. 5. The end point of each curve indicates the failure load and correlates with the quasi-static longitudinal compressive strength $X_{\mathrm{C}}^{\mathrm{qs}}$ and the ultimate failure strain $\epsilon_{\mathrm{C}}^{\mathrm{u}}$. The results of the reference tests, the mean values (Mean), the standard deviation (STDV) and the coefficient of variance (CV) are summarized in Table 1. The mean compressive strength of $604.86 \mathrm{MPa}$ shows a relative high coefficient of variance with $16.30 \%$ but no correlation to varying failure modes could be observed. For all quasi-static reference tests, valid failure occurred in the form of fiber kinking away from the loading surfaces (Fig. 6). The kink-bands for every specimen are marked with yellow arrows respectively (Fig. 6). Three of five specimens lost one bonded tap with ultimate load. The taps fail adhesively with the black adhesive on the glass-epoxy tab (Fig. 6). 


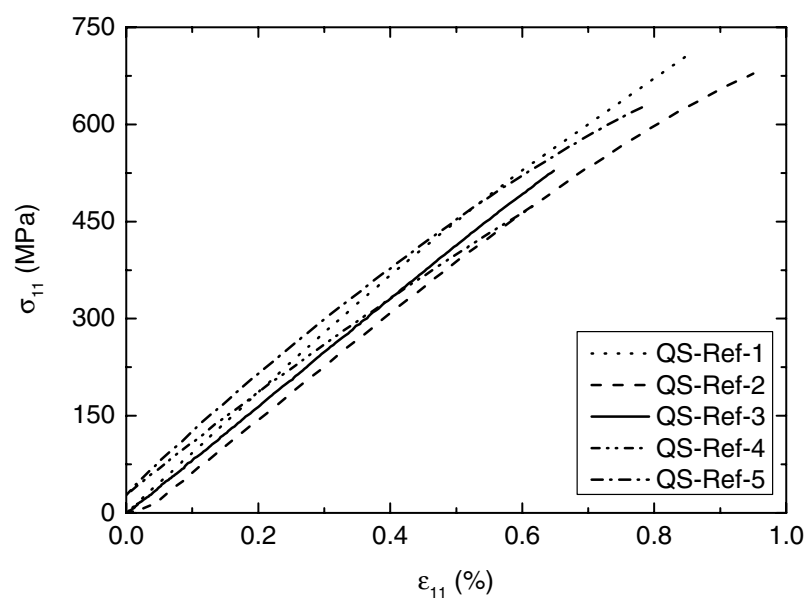

Figure 5. Quasi-static longitudinal compressive stress-strain curves from reference tests.

Table 1. Quasi-static reference test results.

\begin{tabular}{lcc}
\hline Test & $\begin{array}{c}\text { Strength } \\
X_{\mathrm{C}}^{\mathrm{qs}}(\mathrm{MPa})\end{array}$ & $\begin{array}{c}\text { Failure Strain } \\
\epsilon_{\mathrm{C}}^{\mathrm{u}}(\%)\end{array}$ \\
\hline QS-Ref-1 & 708.66 & 0.85 \\
QS-Ref-2 & 678.49 & 0.95 \\
QS-Ref-3 & 528.53 & 0.65 \\
QS-Ref-4 & 477.63 & 0.62 \\
QS-Ref-5 & 630.99 & 0.79 \\
\hline Mean & 604.86 & 0.77 \\
STDV & 98.61 & 0.14 \\
CV $(\%)$ & 16.30 & 17.90 \\
\hline
\end{tabular}

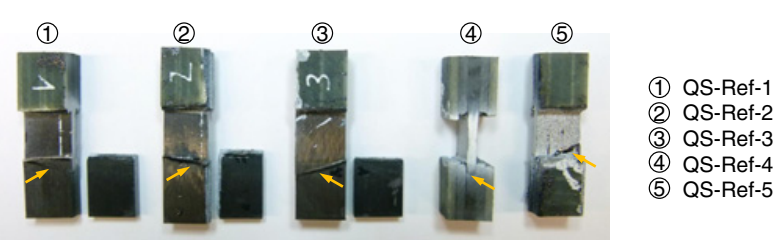

Figure 6. Quasi-static specimen failure mode of reference tests.

\subsection{Dynamic experimental results}

The dynamic test results were calculated by using the classic SHPB analysis, which is based on the onedimensional wave propagation theory [17]. The forces acting at the incident-bar $F_{1}$ and at the transmission-bar $F_{2}$ side of the specimen respectively are defined as

$$
\begin{gathered}
F_{1}=A_{\mathrm{B}} E_{\mathrm{B}}\left(\epsilon_{\mathrm{I}}+\epsilon_{\mathrm{R}}\right) \\
F_{2}=A_{\mathrm{B}} E_{\mathrm{B}} \epsilon_{\mathrm{T}} .
\end{gathered}
$$

Here $\epsilon_{\mathrm{I}}, \epsilon_{\mathrm{R}}$ and $\epsilon_{\mathrm{T}}$ are the incident-, reflected and transmitted bar strains, $A_{\mathrm{B}}$ the bar cross-section and $E_{\mathrm{B}}$ the Young's modulus of the SHPB bars, respectively. It is known that the one-dimensional wave equation can be solved by using the D'Alembert's method and finally describes the specimen strain $\epsilon_{\mathrm{S}}(\mathrm{t})$ and the specimen strain rate $\dot{\epsilon_{\mathrm{S}}}(\mathrm{t})$ as follows:

$$
\begin{gathered}
\epsilon_{\mathrm{S}}(\mathrm{t})=\left(c_{\mathrm{B}} / l_{\mathrm{S}}\right) \int\left(-\epsilon_{\mathrm{I}}(\mathrm{t})+\epsilon_{\mathrm{R}}(\mathrm{t})+\epsilon_{\mathrm{T}}(\mathrm{t})\right) \mathrm{dt} \\
\dot{\epsilon}_{\mathrm{S}}(\mathrm{t})=\left(c_{\mathrm{B}} / l_{\mathrm{S}}\right)\left(-\epsilon_{\mathrm{I}}(\mathrm{t})+\epsilon_{\mathrm{R}}(\mathrm{t})+\epsilon_{\mathrm{T}}(\mathrm{t})\right)
\end{gathered}
$$
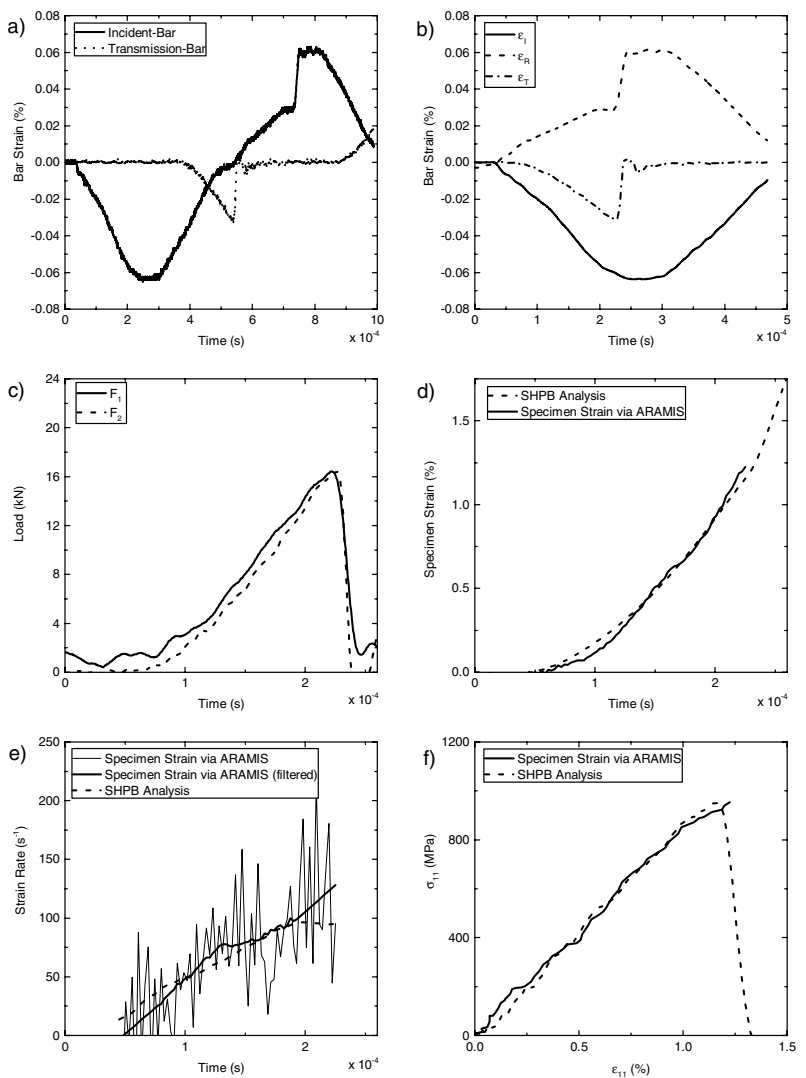

Figure 7. Incident and transmission bar signals of the specimen HR-1 a) and SHPB analysis results of the specimen HR-1 for the shifted bar strain waves b), the loads acting on the specimen c), the specimen strain behavior d), the specimen strain rate e) over time and the stress-strain response f).

where $l_{\mathrm{S}}$ represents the initial specimen length and $c_{\mathrm{B}}$ the elastic wave propagation velocity of the bar material. The wave velocity is calculated by using the equation $c_{\mathrm{B}}=$ $\left(E_{\mathrm{B}} / \rho\right)^{0.5}=5123 \mathrm{~m} / \mathrm{s}$ and the Young's modulus $E_{\mathrm{B}}=$ $206000 \mathrm{MPa}$ and the density $\rho=7850 \mathrm{~kg} / \mathrm{m}^{3}$ of the steel bars used in the SHPB setup. It is noted that in the present work the Eqs. (3) and (4) are only used for comparison because the specimen strain and the specimen strain rate were directly obtained by contactless optical strain measurement via digital image correlation. The specimen stress $\sigma_{\mathrm{S}}$ is then calculated by the quotient of $F_{2}$ and the initial specimen cross-section $A_{\mathrm{S}}$ :

$$
\sigma_{\mathrm{S}}=F_{2} / A_{\mathrm{S}}=\left(A_{\mathrm{B}} E_{\mathrm{B}} \epsilon_{\mathrm{T}}\right) / A_{\mathrm{S}} .
$$

This parameter is also denoted as the 1-wave-stess or back-stress, because it represents the condition at the specimen/transmission-bar interface.

Figure 7 shows the post-processing results of the bar strain waves for a representative specimen (specimen number: HR-1). The graphs serve as illustration of the SHPB analysis. The unfiltered incident- and transmissionbar signals are shown in Fig. 7a). As a first step the bar strain waves detected at the two strain gauges on the incident- and transmission-bar are filtered with a SavitzkyGolay filter and then shifted to the time at which they occurred at the specimen (Fig. $7 \mathrm{~b})$ ). The loads $F_{1}$ and $F_{2}$ were calculated with the Eqs. (1) and (2) and plotted over 


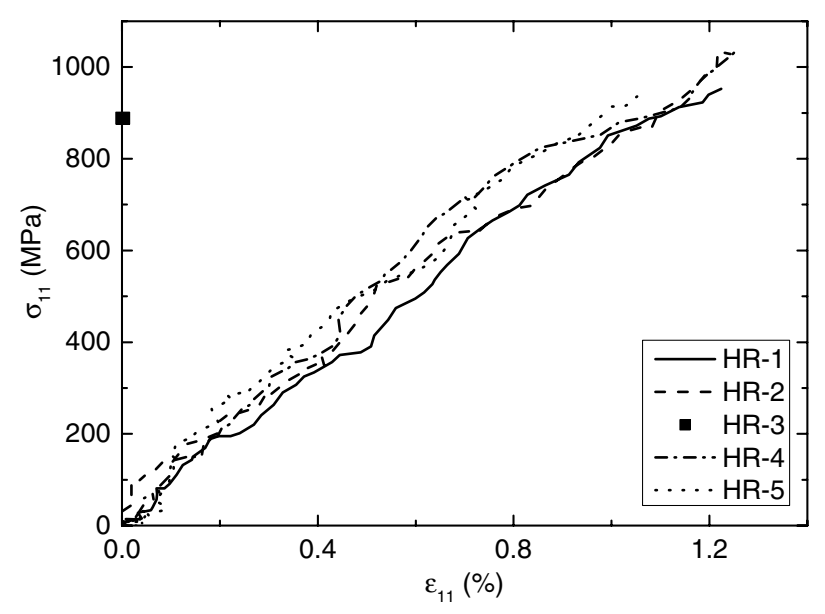

Figure 8. Dynamic longitudinal compressive stress-strain response of all tested specimens.

time (Fig. 7c)). The reason for the initial offset of the forces is the nonzero bar strain at the beginning of the reflected signal. Taking this into account, the load-time responses on both sides of the specimen are similar and thus dynamic equilibrium during the experiment could be presumed. A comparison of the specimen strain calculated with Eq. (3) and the specimen strain measured optically by using the ARAMIS system is shown in Fig. 7d). Both curves are very similar, but Koerber and Camanho [14] already showed that this is not always the case. Accompanied with the strain-time responses, the strain rate-time responses obtained from Eq. (4) and the slope of the ARAMIS strain signal, were compared (Fig. 7e)). Due to the small noise of the ARAMIS strain signal the strain rate signal oscillates significantly. The strain rate signal is filtered with a Savitzky-Golay filter. A constant strain rate could be achieved after half of the experiment. In Fig. 7f) the calculated specimen strain and the optically measured strain are plotted as a function of the specimen stress calculated with the Eq. (5).

The longitudinal compressive stress-strain response of all dynamically tested specimens (Fig. 8) is based on the optical measured strain and the calculated stress via the SHPB analysis. Due to the digitalization of the bar strain signals and the discrete number of strain values received from the digital image correlation the stress-strain curves are somewhat noisy. The failure load is indicated at the end point of each curve and corresponds to the longitudinal compressive strength $X_{C}^{\mathrm{hr}}$ and the ultimate failure strain $\epsilon_{\mathrm{C}}^{\mathrm{u}}$. For specimen HR-3 only the failure load was recorded because of a non-functioning high-speed camera record. In Table 2 all dynamic experimental results are listed. A mean compressive strength of $974.93 \mathrm{MPa}$ with a coefficient of variance of $6.47 \%$ for the unidirectional carbon fiber reinforced polyamide- 6 composite was measured. All specimens fail similar to the quasi-static specimens away from the loading surface (Fig. 9) due to the formation of kink-band followed by through thickness fiber failure. The pictures of the high speed camera show, that with maximum stress the initiation of the fiber kinking starts and subsequently the development of the kink-band. The delamination of the base-laminate in the region of the
Table 2. Dynamic test results.

\begin{tabular}{cccc}
\hline Test & $\begin{array}{c}\text { Strength } \\
X_{\mathrm{C}}^{\mathrm{hr}}(\mathrm{MPa})\end{array}$ & $\begin{array}{c}\text { Failure Strain } \\
\epsilon_{\mathrm{C}}^{\mathrm{u}}(\%)\end{array}$ & $\begin{array}{c}\text { Strain Rate } \\
\dot{\epsilon}\left(\mathrm{s}^{-1}\right)\end{array}$ \\
\hline HR-1 & 952.88 & 1.22 & 96.05 \\
HR-2 & 1034.52 & 1.22 & 85.93 \\
HR-3 & 888.05 & - & - \\
HR-4 & 1039.13 & 1.26 & 110.72 \\
HR-5 & 960.05 & 1.06 & 92.56 \\
\hline Mean & 974.93 & 1.19 & 96.32 \\
STDV & 63.10 & 0.09 & 10.48 \\
CV $(\%)$ & 6.47 & 7.45 & 10.88 \\
\hline
\end{tabular}

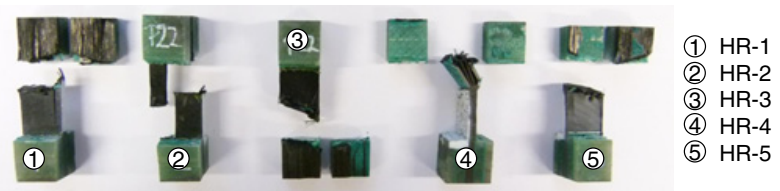

Figure 9. Dynamic specimen failure mode.

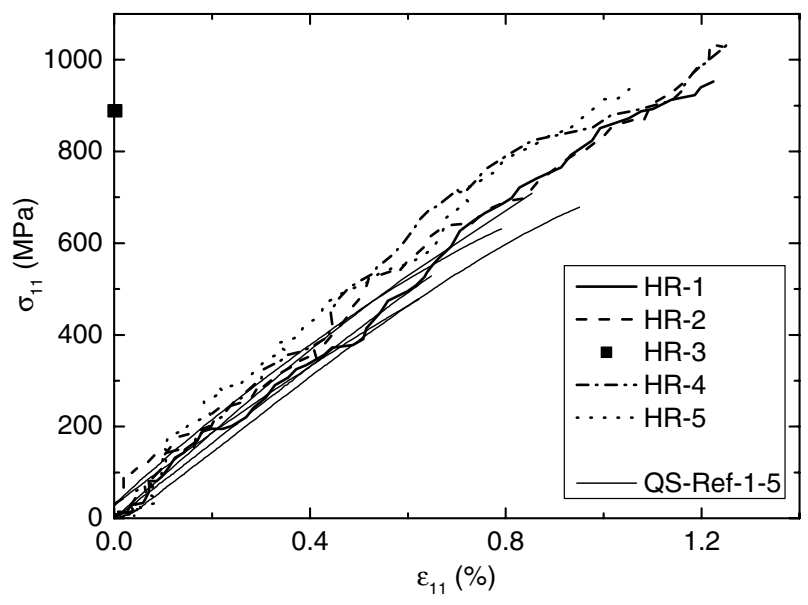

Figure 10. Comparison of the quasi-static longitudinal compressive stress-strain curves from reference and dynamic tests.

glass-epoxy tabs occurred after the formation of the kinkband and additional longitudinal cracks.

The comparison of all quasi-static reference test results and all dynamic test results indicates that the longitudinal compressive strength of the unidirectional carbon fiber reinforced polyamide- 6 composite increased by $61 \%$ (Fig. 10). The Young's Modulus of the material at high strain rate shows a minor increase with increasing strain rate.

\section{Conclusion}

The strain rate dependency of the longitudinal compressive stress-strain response of a unidirectional carbon fiber reinforced polyamide- 6 composite was investigated in the presented work. It was proven that the developed end-loaded compression specimen geometry, consisting of a flat rectangular unidirectional base-laminate and glass-epoxy tabs, is suitable for dynamic loading in a spit-Hopkinson pressure bar and additionally useful for contactless optical strain measurement via digital image correlation. Quasi-static reference tests were carried out at a strain rate of approximately $3 \cdot 10^{-4} \mathrm{~s}^{-1}$ using a 
self-aligning loading device with embedded polished tungsten-carbide inserts at the loading surfaces. For all quasi-static tests valid failure modes in form of fiber kinking away from the loading surfaces were observed. For the high strain rate tests the Dynamic Compression Fixture [14] was modified to provide a view field for the optical strain measurement via a high-speed camera to monitor the specimen deformation and failure mode. Similar to the quasi-static reference tests, all dynamic specimens failed due to fiber kinking away from the loading surfaces. It was observed that the longitudinal compressive strength of the unidirectional carbon fiber reinforced polyamide- 6 composite increased by $61 \%$ compared to the strength value obtained from the quasi-static reference tests.

The authors would like to acknowledge the German Federal Ministry of Education and Research (BMBF) for providing funding within the project MAI Plast.

\section{References}

[1] M. Biron, Thermoplastics and thermoplastic composites, 1st edn. (Butterworth-Heinemann, Oxford, 2007)

[2] E. Baur, S. Brinkmann, T. Osswald, E. Schmachtenberg, Saechtling Kunststoff Taschenbuch, 30th edn. (Carl Hanser Verlag, München, 2007)

[3] L. Bottenbruch, R. Binsack, G. Becker, D. Braun, Polyamide. Kunststoffhandbuch 3/4. (Hanser, 1998)

[4] S.M. Walley, J.E. Field, DYMAT Journal 1, 211-227 (1994)
[5] H. Pouriayevali, S. Arabnejad, Y.B. Guo, V.P.W. Shim, International Journal of Impact Engineering 62, 35-47 (2013)

[6] G.-F. Shan, W. Yang, M. Yang, B. Xie, J. Feng, Q. Fu, Polymer 48, 2958-2968 (2007)

[7] M. Hokka, V.-T. Kuokkala, S. Ihme, Proceedings of the SEM Annual Conference June 1-4, 2009 Albuquerque New Mexico USA, Society for Experimental Mechanics Inc. (2009)

[8] A. Vashchenko, I. Spiridonova, E. Sukhovaya, Metalurgija 39, 89-92 (2000)

[9] Z. Wang, Y. Zhou, P.K. Mallick, Polymer Composites 23, 858-871 (2002)

[10] B. Mouhmid, A. Imad, N. Benseddiq, S. Benmedakhène, A. Maazouz, Polymer Testing 25, 544-552 (2006)

[11] S.J.H. Kuhlman, S.I. Hill, SAE Technical Paper 2014-01-1056 (2014)

[12] M. Todo, K. Takahashi, P. Béguelin, H.H. Kausch, Composites Science and Technology 60, 763-771 (2000)

[13] Z. Jendli, J.-C. Walrick, M. Bocquet, J. Fitoussi, Proceedings of ECCM16 - 16th European Conference on Composite Materials, Seville, Spain (2014)

[14] H. Koerber, P.P. Camanho, Composites Part A 42, 462-470 (2011)

[15] Material Data Sheet: Standard Product Values Celstran CFR-TP PA6 CF60-01 (7/29/2014)

[16] ASTM D3171: Standard test methods for constituent content of composite materials (1999)

[17] G.T. Gray III, ASM Handbook Vol. 8 Mechanical Testing and Evaluation (ASM International, Ohio, 2000) 462-476 